\title{
POWER SERIES SOLUTION OF FIRST ORDER MATRIX DIFFERENTIAL EQUATIONS
}

\author{
Stanisław Kukla, Izabela Zamorska \\ Institute of Mathematics, Czestochowa University of Technology \\ Częstochowa, Poland \\ stanislaw.kukla@im.pcz.pl,izabela.zamorska@im.pcz.pl
}

\begin{abstract}
In the paper, the solution of second order differential equations with various coefficients is presented. The concerning equations are written as first order matrix differential equations and solved with the use of the power series method. Examples of application of the proposed method to the equations occurring in the technical problems are presented.
\end{abstract}

Keywords: power series, differential equations

\section{Introduction}

The goal of many researchers' work is finding new and improving existing various methods for solving ordinary differential equations. In various types of differential equations (linear and non-linear, with constant or variable coefficients), occurring, for example, in technical specification number of issues, it is possible to designate the exact solution analysis, and in the others, however, it is necessary to approximate methods. One of the classical methods, known since the seventeenth century, in solving differential equations is the method of power series, involving the appointment of a solution in the form of an infinite Taylor series (series coefficients are obtained from recursive equations). This method was applied e.g. by Eisenberger in [1] to solve the second order differential equation describing rods vibration problems and by Quaisi in [2] for the non-linear free vibration of beams with restrained ends. In turn, Zhou in [3] proposed a method of differential transformation - an improved method of power series, differing the way of determining the coefficients of the series. A power series method with domain partition in an implemented matrix formulation is another method, alternative to other techniques of power series [4].

In this paper, the method of solving second order ordinary differential equation will be presented by transforming this equation in the system of differential equations of the first order, then presenting it in matrix notation and solving with the use of power series method. As examples there will be presented solutions of two classic equations: the Airy equation and equation occurring in the description of the rod's vibration. 


\section{Formulation and solution of the problem}

Let us consider the first order matrix differential equation

$$
\mathbf{Y}^{\prime}(x)=\mathbf{B}(x) \mathbf{Y}(x)+\mathbf{F}(x)
$$

where $\mathbf{Y}(x)=\left[\begin{array}{llll}y_{1}(x) & y_{2}(x) & \cdots & y_{m}(x)\end{array}\right]^{T}, \quad \mathbf{F}(x)=\left[\begin{array}{lllll}f_{1}(x) & f_{2}(x) & \cdots & f_{m}(x)\end{array}\right]^{T}$ and $\mathbf{B}(x)=\left[b_{i j}(x)\right]_{1 \leq i, j \leq m}$. The equation is completed by initial condition

$$
\mathbf{Y}(0)=\mathbf{Y}_{0}
$$

We are looking on solution of the equation (1) in the form of power series $\mathbf{Y}(x)=\sum_{n=0}^{\infty} \mathbf{Y}_{n} \frac{x^{n}}{n !}$ where $\mathbf{Y}_{n}=\left[\begin{array}{llll}y_{n 1} & y_{n 2} & \cdots & y_{n m}\end{array}\right]^{T}$. Assuming that $\mathbf{B}(x)=\sum_{n=0}^{\infty} \mathbf{B}_{n} \frac{x^{n}}{n !}$ and $\mathbf{F}(x)=\sum_{n=0}^{\infty} \mathbf{F}_{n} \frac{x^{n}}{n !}$, substituting into equation (1) we obtain:

$$
\left(\sum_{n=0}^{\infty} \mathbf{Y}_{n} \frac{x^{n}}{n !}\right)^{\prime}=\left(\sum_{n=0}^{\infty} \mathbf{B}_{n} \frac{x^{n}}{n !}\right)\left(\sum_{n=0}^{\infty} \mathbf{Y}_{n} \frac{x^{n}}{n !}\right)+\sum_{n=0}^{\infty} \mathbf{F}_{n} \frac{x^{n}}{n !}
$$

After transformations in equation (3) we get the following recursive relation

$$
\begin{gathered}
\mathbf{Y}_{1}=\mathbf{F}_{0}+\mathbf{B}_{0} \mathbf{Y}_{0} \\
\mathbf{Y}_{n+1}=\mathbf{F}_{n}+\sum_{k=0}^{n}\left(\begin{array}{l}
n \\
k
\end{array}\right) \mathbf{B}_{k} \mathbf{Y}_{n-k} \quad n=1,2, \ldots
\end{gathered}
$$

The coefficients $\mathbf{Y}_{n}$ may be written as:

$$
\mathbf{Y}_{n}=\Phi_{n}+\Psi_{n} \mathbf{Y}_{0} \quad n=0,1,2, \ldots
$$

where $\Phi_{0}=\mathbf{0}, \quad \Psi_{0}=\mathbf{E} \quad$ (the unit matrix) and $\Phi_{n+1}=\mathbf{F}_{n}+\sum_{k=0}^{n}\left(\begin{array}{l}n \\ k\end{array}\right) \mathbf{B}_{n-k} \Phi_{k}$, $\Psi_{n+1}=\sum_{k=0}^{n}\left(\begin{array}{l}n \\ k\end{array}\right) \mathbf{B}_{n-k} \Phi_{k}$.

Finally, a solution of an inhomogeneous matrix differential equation (1) can be expressed as a sum

$$
\mathbf{Y}(x)=\sum_{n=0}^{\infty} \Phi_{n} \frac{x^{n}}{n !}+\left(\sum_{n=0}^{\infty} \Psi_{n} \frac{x^{n}}{n !}\right) \mathbf{Y}_{0}=\Phi_{n}(x)+\Psi_{n}(x) \mathbf{Y}_{0}
$$




\section{Examples of method's application}

Let the ordinary differential equation of second order with variable coefficients

$$
\frac{d^{2} y(x)}{d x^{2}}+a_{2}(x) \frac{d y(x)}{d x}+a_{1}(x) y(x)=f(x)
$$

with the conditions:

$$
y(0)=y_{0}, \quad y(l)=y_{l}
$$

Introducing functions $y_{1}(x)=y(x), y_{2}(x)=y_{1}^{\prime}(x)$, equation (7) can be written as a system

$$
\begin{gathered}
y_{1}^{\prime}(x)=y_{2}(x) \\
y_{2}^{\prime}(x)=f(x)-a_{1} y_{1}(x)-a_{2} y_{2}(x)
\end{gathered}
$$

or in the matrix form as equation (1) where $\mathbf{Y}(x)=\left[\begin{array}{ll}y_{1}(x) & y_{2}(x)\end{array}\right]^{T}$, $\mathbf{F}(x)=\left[\begin{array}{ll}0 & f(x)\end{array}\right]^{T}$ and $\mathbf{B}(x)=\left[\begin{array}{cc}0 & 1 \\ -a_{1}(x) & -a_{2}(x)\end{array}\right]$.

Functions occurring in equation (7) are $C^{\infty}$ class and may be written as

$$
y_{i}(x)=\sum_{n=0}^{\infty} y_{i n} \frac{x^{n}}{n !}, a_{i}(x)=\sum_{n=0}^{\infty} a_{i n} \frac{x^{n}}{n !}(i=1,2), f(x)=\sum_{n=0}^{\infty} f_{n} \frac{x^{n}}{n !}
$$

Initial and boundary equations ( 8 ) are as follows:

$$
\mathbf{Y}_{0}=\left[\begin{array}{l}
y_{1}(0) \\
y_{2}(0)
\end{array}\right], \mathbf{Y}_{l}=\left[\begin{array}{l}
y_{1}(l) \\
y_{2}(l)
\end{array}\right]
$$

where $y_{1}(0)=y_{0}, y_{1}(l)=y_{l}, y_{2}(0)$ and $y_{2}(l)$ can be, respectively, determined from the relations

$$
y_{2}(0)=y_{11}=\frac{1}{l}\left(y_{l}-y_{0}+\sum_{n=2}^{\infty} y_{1 n} \frac{l^{n}}{n !}\right), y_{2}(l)=\sum_{n=0}^{\infty} y_{1 n+1} \frac{l^{n}}{n !} .
$$

\section{Airy equation}

Schrödinger equation, one of the fundamental equations of non-relativistic quantum mechanics, under certain assumptions can be reduced to the Airy equation 


$$
y^{\prime \prime}(x)+x y(x)=0
$$

which has a well-known analytical solution in the form:

$$
y(x)=\frac{\sqrt{x}}{3}\left[C_{1} J_{-\frac{1}{3}}\left(\frac{2}{3} \sqrt{x^{3}}\right)+C_{2} J_{\frac{1}{3}}\left(\frac{2}{3} \sqrt{x^{3}}\right)\right]
$$

where $J(\cdot)$ is a Bessel function of the first kind and constant values $C_{1}, C_{2}$ depend on the selected initial conditions.

Using the matrix notation we have

$$
\mathbf{F}(x)=\mathbf{0}, \mathbf{B}(x)=\sum_{n=0}^{\infty} \mathbf{B}_{n} \frac{x^{n}}{n !}=\mathbf{B}_{0}+\mathbf{B}_{1} x
$$

where $\mathbf{B}_{0}=\left[\begin{array}{ll}0 & 1 \\ 0 & 0\end{array}\right], \mathbf{B}_{1}=\left[\begin{array}{cc}0 & 0 \\ -1 & 0\end{array}\right], \mathbf{B}_{2}=\mathbf{B}_{3}=\ldots=\mathbf{0}$. Recursive relations for series coefficients $\mathbf{Y}_{n+1}$ are

$$
\mathbf{Y}_{1}=\mathbf{B}_{0} \mathbf{Y}_{0}, \quad \mathbf{Y}_{n+1}=\mathbf{B}_{0} \mathbf{Y}_{n}+n \mathbf{B}_{1} \mathbf{Y}_{n-1} \quad n=1,2, \ldots
$$

Solution of Airy equation (13) in a matrix form may be written as

$$
\mathbf{Y}(x)=\left(\sum_{n=0}^{\infty} \Psi_{n} \frac{x^{n}}{n !}\right) \mathbf{Y}_{0}
$$

where the first few values of $\Psi_{n}$ are:

$$
\begin{aligned}
& \Psi_{0}=\mathbf{E} \\
& \Psi_{1}=\mathbf{B}_{0} \\
& \Psi_{2}=\mathbf{B}_{0}^{2}+\mathbf{B}_{1} \\
& \Psi_{3}=\mathbf{B}_{0}^{3}+\mathbf{B}_{0} \mathbf{B}_{1}+2 \mathbf{B}_{1} \mathbf{B}_{0} \\
& \Psi_{4}=\mathbf{B}_{0}^{4}+\mathbf{B}_{0}^{2} \mathbf{B}_{1}+2 \mathbf{B}_{0} \mathbf{B}_{1} \mathbf{B}_{0}+3 \mathbf{B}_{1} \mathbf{B}_{0}^{2}+3 \mathbf{B}_{1}^{2} \\
& \Psi_{5}=\mathbf{B}_{0}^{5}+\mathbf{B}_{0}^{3} \mathbf{B}_{1}+2 \mathbf{B}_{0}^{2} \mathbf{B}_{1} \mathbf{B}_{0}+3 \mathbf{B}_{0} \mathbf{B}_{1} \mathbf{B}_{0}^{2}+3 \mathbf{B}_{0} \mathbf{B}_{1}^{2}+4 \mathbf{B}_{1} \mathbf{B}_{0}^{3}+4 \mathbf{B}_{1} \mathbf{B}_{0} \mathbf{B}_{1}+8 \mathbf{B}_{1}^{2} \mathbf{B}_{0}
\end{aligned}
$$

By using designations:

$\mathbf{M}_{0}=\left[\begin{array}{ll}1 & 0 \\ 0 & 1\end{array}\right], \mathbf{M}_{1}=\left[\begin{array}{ll}1 & 0 \\ 0 & 0\end{array}\right], \mathbf{M}_{2}=\left[\begin{array}{ll}0 & 1 \\ 0 & 0\end{array}\right], \mathbf{M}_{3}=\left[\begin{array}{ll}0 & 0 \\ 1 & 0\end{array}\right], \mathbf{M}_{4}=\left[\begin{array}{ll}0 & 0 \\ 0 & 1\end{array}\right]$

$\left(\mathbf{B}_{0}=\mathbf{M}_{2}, \mathbf{B}_{1}=-\mathbf{M}_{3}\right)$, it can be seen that sets of matrices $\left\{\mathrm{O}, \mathbf{M}_{0}, \mathbf{M}_{1}, \mathbf{M}_{2}, \mathbf{M}_{3}, \mathbf{M}_{4}\right\}$ with sets multiplication create a semigroup. The algebraic properties of pair ( $\left\{\mathrm{O}, \mathbf{M}_{0}, \mathbf{M}_{1}, \mathbf{M}_{2}, \mathbf{M}_{3}, \mathbf{M}_{4}\right\}, \times$ ) describes the multiplication table (Table 1). 
Multiplication table

Table 1

\begin{tabular}{|c|c|c|c|c|c|}
\hline$\times$ & $\mathrm{M}_{0}$ & $\mathrm{M}_{1}$ & $\mathrm{M}_{2}$ & $\mathrm{M}_{3}$ & $\mathrm{M}_{4}$ \\
\hline $\mathrm{M}_{0}$ & $\mathrm{M}_{0}$ & $\mathrm{M}_{1}$ & $\mathrm{M}_{2}$ & $\mathrm{M}_{3}$ & $\mathrm{M}_{4}$ \\
\hline $\mathrm{M}_{1}$ & $\mathrm{M}_{1}$ & $\mathrm{M}_{1}$ & $\mathrm{M}_{2}$ & $\mathrm{O}$ & $\mathrm{O}$ \\
\hline $\mathrm{M}_{2}$ & $\mathrm{M}_{2}$ & $\mathrm{O}$ & $\mathrm{O}$ & $\mathrm{M}_{1}$ & $\mathrm{M}_{2}$ \\
\hline $\mathrm{M}_{3}$ & $\mathrm{M}_{3}$ & $\mathrm{M}_{3}$ & $\mathrm{M}_{4}$ & $\mathrm{O}$ & $\mathrm{O}$ \\
\hline $\mathrm{M}_{4}$ & $\mathrm{M}_{4}$ & $\mathrm{O}$ & $\mathrm{O}$ & $\mathrm{M}_{3}$ & $\mathrm{M}_{4}$ \\
\hline
\end{tabular}

$$
\text { Equation } \quad \frac{d}{d x}\left(p(x) \frac{d y}{d x}\right)+k p(x) y=0
$$

This type of equation occurs frequently in problems of mechanics, for example in the vibration's description of rods or strings. The functions occurring in the equation have a physical interpretation: $y(x)$ is a function of deflection, $p(x)$ is a cross-section area and $\sqrt{k}$ is a parameter characterized vibration frequency of the mechanical system under consideration.

Suppose that $p(x)=e^{-\alpha x}$ where $\alpha$ is a constant, $x \in\langle 0,1\rangle$. The general solution of homogeneous equation (18) is [5]

$$
y(x)=e^{\frac{\alpha}{2} x}\left[C_{1} \cos \left(\frac{x}{2} \sqrt{4 k-\alpha^{2}}\right)+C_{2} \sin \left(\frac{x}{2} \sqrt{4 k-\alpha^{2}}\right)\right]
$$

Matrices $\mathbf{B}$ and $\mathbf{F}$ existing in the equation (1) are as follows:

$$
\begin{gathered}
\mathbf{B}(x)=\left[\begin{array}{cc}
0 & 1 \\
-k & \frac{-p^{\prime}(x)}{p(x)}
\end{array}\right]=\left[\begin{array}{cc}
0 & 1 \\
-k & \alpha
\end{array}\right]=\mathbf{B}_{0}, \quad \mathbf{B}_{n}=\mathbf{0} \quad n=1,2, \ldots \\
\mathbf{F}(x)=\mathbf{0}
\end{gathered}
$$

Series coefficients of the solution of (18) are:

$$
\mathbf{Y}_{0}, \mathbf{Y}_{1}=\mathbf{B}_{0} \mathbf{Y}_{0}, \mathbf{Y}_{2}=\mathbf{B}_{0}^{2} \mathbf{Y}_{0}, \ldots, \mathbf{Y}_{n}=\mathbf{B}_{0}^{n} \mathbf{Y}_{0}
$$

and the solution may be written in the form:

$$
\mathbf{Y}(x)=\left(\sum_{n=0}^{\infty} \mathbf{B}_{0}^{n} \frac{x^{n}}{n !}\right) \mathbf{Y}_{0}
$$




\section{Conclusions}

The method of solving second order linear differential equations, presented in the work, may be used for solving $n$-th order linear equations. Any of these equations can be represented by a system of $n$ first order linear differential equations with more than one dependent variable and, as the result, by one first order differential matrix equation. Although this method shouldn't be applied indiscriminately, it is suitable to solve, particularly, equations of arbitrary order with variable coefficients which typically arise in vibration or heat transfer problems.

\section{References}

[1] Eisenberger M., Exact longitudinal vibration frequencies of a variable cross-section rods, Applied Acoustics 1991, 34, 123-130.

[2] Quaisi M.I., A power series solution for the non-linear vibration of beams, Journal of Sound and Vibration 1997, 199(4), 587-594.

[3] Zhou K., Differential Transformation and Its Application to Electrical Circuits, Wuhan People Republic of China, Huazhong University Press 1986 (in Chinese).

[4] Inaudi J.A., Matusevich A.E., Domain-partition power series in vibration analysis of variable cross-section rods, Journal of Sound and Vibration 2010, 329, 4534-4549.

[5] Zamorska I., Longitudinal vibrations of a non-uniform rods coupled by double spring-mass systems, Scientific Research of the Institute of Mathematics and Computer Science 2007, 1(6), 277-284. 